\title{
Electro-Acupuncture Alleviates Cisplatin-Induced Anorexia in Rats by Modulating Ghrelin and Monoamine Neurotransmitters
}

\author{
Ji Yun Baek ${ }^{1,2,+}{ }^{\oplus}$, Tuy An Trinh ${ }^{1,+}$, Wonsang Huh ${ }^{1,+}$, Ji Hoon Song ${ }^{1}{ }^{\oplus}$, Hyun Young Kim ${ }^{2}$, \\ Juhee Lim ${ }^{3}$, Jinhee Kim ${ }^{3}$, Hyun Jin Choi ${ }^{3}$, Tae-Hun Kim ${ }^{4, *}$ and Ki Sung Kang ${ }^{1, *}$ \\ 1 College of Korean Medicine, Gachon University, Seongnam 13120, Korea; wldbsttn@naver.com (J.Y.B.); \\ tuyan2308@gc.gachon.ac.kr (T.A.T.); wshuh@gc.gachon.ac.kr (W.H.); jhsong.john@gmail.com (J.H.S.) \\ 2 Department of Food Science, Gyeongnam National University of Science and Technology, Jinju 52725, Korea; \\ hykim@gntech.ac.kr \\ 3 College of Pharmacy and Institute of Pharmaceutical Sciences, CHA University, Seongnam 13488, Korea; \\ juheelim@cha.ac.kr (J.L.); kjh05267@naver.com (J.K.); hjchoi3@cha.ac.kr (H.J.C.) \\ 4 Korean Medicine Clinical Trial Center, Korean Medicine Hospital, Kyung Hee University, Seoul 02447, Korea \\ * Correspondence: rockandmineral@gmail.com (T.-H.K.); kkang@gachon.ac.kr (K.S.K.); \\ Tel.: +82-2-958-9088 (T.-H.K.); +82-031-750-5426 (K.S.K.) \\ + These authors contributed equally to the work described in this study.
}

Received: 12 September 2019; Accepted: 15 October 2019; Published: 18 October 2019

check for updates

\begin{abstract}
Anorexia is common in patients with cancer, mostly as a side effect of chemotherapy. The effect of electro-acupuncture (EA) on ameliorating cancer-related symptoms have been studied in animal models and in clinical trials. The aim of this study was to determine optimal conditions for the application of EA to alleviate anorexia, followed by the study of molecular mechanisms affecting its therapeutics. Anorexia was induced in male Wistar rats by injecting cisplatin, which was then followed by EA treatment at CV12, the acupuncture point located in the center of the abdominal midline. Body weight and food intake were measured daily throughout the duration of the study. The levels of monoamine neurotransmitters in the plasma were quantitatively analyzed by HPLC-ECD. Gastrointestinal hormone concentrations were elucidated with ELISA kits. RT-qPCR was performed to evaluate the mRNA expression of ghrelin (GHRL), neuropeptide Y (NPY), and pro-opiomelanocortin. The expression of c-Fos in the nucleus tractus solitarii was detected using western blotting analysis. The optimal conditions of EA to alleviate anorexia in rats was determined to be 1 unit for intensity and $10 \mathrm{~Hz}$ for frequency. EA treatment at CV12 reduced the levels of plasma monoamine neurotransmitters 5-hydroxytryptamine, 5-hydroxyindoleacetic acid, dopamine, and norepinephrine; as well as stimulated the expression of GHRL and NPY to alleviate cisplatin-induced anorexia in rats. EA stimulation at CV12 could be used to treat cisplatin-induced anorexia in rats.
\end{abstract}

Keywords: electro-acupuncture; 5-hydroxytryptamine; ghrelin; neuropeptide Y; pro-opiomelanocortin; cisplatin; anorexia; cancer

\section{Introduction}

Cancer is a major cause of death in Korea, accounting for $27.8 \%$ of total mortality in 2016 [1]. The forecast for newly diagnosed cases of cancer in Korea for 2019 is 221,347 [2]. Besides cancer developing to advanced stages, malnutrition of patients also contributes to mortality [3]. Anorexia is commonly seen in patients with cancer, especially those undergoing chemotherapy. Anorexia is defined as the loss of interest in food, expressed through loss of appetite, early satiety, or alterations in 
the taste and odor of food [4]. Anorexia is normally accompanied by cachexia, which causes weight loss, mainly from adipose tissues and skeletal muscles [3]. Approximately $65 \%$ of terminally ill patients with cancer suffer from anorexia [5]. It leads to weakness of the body and affects the recovery capacity, as well as the survival rate of those affected.

Anorexia is associated with the development of cancer, like a physiological response to tumor growth. Aside from anorexia, nausea and vomiting are also common side effects of chemotherapy and radiotherapy [6]. Multiple factors contribute to the pathogenesis of anorexia in cancer through hypothalamic neuronal signaling pathways such as gastrointestinal hormones, energy signals, neurotransmitters, neuropeptides, and hypothalamic neuroimmune interactions [5,6]. Therapeutic strategies to treat anorexia related to cancer focus on improving appetite and neutralizing metabolic disorders. The common drugs used to stimulate appetite are progestins, cannabinoids, cyproheptadine, corticosteroids, GHRL, and melanocortin antagonists [5-7].

Oriental medicine is commonly used as an adjunctive therapy for the treatment of cancer. Along with herbal medicine, acupuncture may be used to alleviate the side effects of anticancer therapies. Acupuncture has been practiced in Asian countries for thousands of years. More recently, various methods of acupuncture have been developed such as manual acupuncture, EA, transcutaneous electric nerve stimulation, acupressure, auricular acupuncture, and moxibustion. Clinical trials have suggested that EA was able to reduce acute vomiting caused by chemotherapy [8,9], improve insomnia [10], and relieve pancreatic pain caused by cancer [11].

The effect of the underlying mechanisms of EA on ameliorating cancer-related symptoms has been studied in animal models. EA at CV12 alleviated cisplatin-induced nausea in rats by reducing the 5-HT level in the duodenum along with inhibiting the expression of c-Fos in the NTS [12]. EA at ST36 decreased the gastrointestinal hormone levels of GHRL, peptide YY, and glucagon-like peptide-1, which contribute to the ameliorating effect on cisplatin-induced dyspepsia in rats [13]. Previously, the therapeutic effects of EA on cisplatin-induced anorexia in rats were evaluated using various acupuncture points such as CV12, PC6, and ST36 [14]. This study aims to ascertain the optimal conditions for EA treatment to alleviate anorexia using evidence from molecular mechanism studies for support.

\section{Materials and Methods}

\subsection{Animals}

Male Wistar rats (body weight, 200-240 g; age, 7 weeks old) were procured from Orient Bio Co., Ltd. (Seongnam, Korea) and used in this study.

All procedures involving the use of live animals described in the present study were approved by the Institutional Animal Care and Use Committee of Gachon University (Seongnam, Korea; approval no. GIACUC-R2015011) in December 2015. The rats were housed in normal conditions and were exposed to a $12 \mathrm{~h} \mathrm{light/dark} \mathrm{cycle} \mathrm{at} 23 \pm 2{ }^{\circ} \mathrm{C}$ and $55 \pm 5 \% \mathrm{RH}$. The rats were fed a standard chow diet containing $10 \%$ fat ad libitum for one week before the experiments were performed.

\subsection{Description of Study}

This study included two experiments, one to evaluate the effects of different EA conditions on cisplatin-induced anorexia, and another to study the pharmacological mechanisms of the optimal EA conditions obtained from the result of the first experiment. In the first experiment, two tests were designed to ascertain for the optimal intensity and frequency of EA conditions. For the intensity study, the rats were randomly divided into 3 groups $(n=3)$ : Cisplatin (injected with cisplatin, without EA), Cisplatin + CV12 (1 unit) (injected with cisplatin, EA at the intensity of 1 unit), and Cisplatin + CV12 (4 units) (injected with cisplatin, EA at the intensity of 4 units). Similarly, the rats were randomly divided into 3 groups $(n=3)$ for the frequency study as well: Cisplatin (injected with cisplatin, without EA), Cisplatin + CV12 $(10 \mathrm{~Hz})$ (injected with cisplatin, EA at the frequency of $10 \mathrm{~Hz})$, and Cisplatin + 
CV12 $(100 \mathrm{~Hz})$ (injected with cisplatin, EA at the frequency of $100 \mathrm{~Hz})$. The effect of each EA condition was assessed through the change in body weight and food intake.

Based on the results obtained from the first experiment, EA at frequency $10 \mathrm{~Hz}$ and intensity 1 unit was chosen for the second part of the experiment. The rats were grouped into $3(n=3)$ : Vehicle (injected with saline; EA intensity 1 unit, frequency $10 \mathrm{~Hz}$ at the non-acupoint), Cisplatin (injected with cisplatin; without EA), and Cisplatin + CV12 (injected with cisplatin; EA intensity 1 unit, frequency $10 \mathrm{~Hz}$ at the CV12 acupoint). After 3 days, the rats were sacrificed and the blood, duodenum, hypothalamus, and brain stems were collected for further analysis. The details of the experimental design are mentioned in Table 1.

Table 1. Evaluation of the effects of electro-acupuncture (EA) on a cisplatin-induced anorexic model.

\begin{tabular}{|c|c|c|c|c|c|}
\hline Experimental Condition & Day -1 & Day 0 & Day 1 & Day 2 & Day 3 \\
\hline \multicolumn{6}{|c|}{ 1st Experiment|Intensity of EA } \\
\hline Cisplatin, i.p. & - & + & - & - & - \\
\hline EA (low intensity stimulation) & - & - & + & + & + \\
\hline EA (high intensity stimulation) & - & - & + & + & + \\
\hline Body weight and food intake & + & + & + & + & + \\
\hline \multicolumn{6}{|c|}{ 1st Experiment|Frequency of EA } \\
\hline Cisplatin, i.p. & - & + & - & - & - \\
\hline EA $(10 \mathrm{~Hz})$ & - & - & + & + & + \\
\hline EA $(100 \mathrm{~Hz})$ & - & - & + & + & + \\
\hline Body weight and food intake & + & + & + & + & + \\
\hline \multicolumn{6}{|c|}{ 2nd Experiment|Pharmacological effects of the optimal EA conditions } \\
\hline Cisplatin, i.p. & - & + & - & - & - \\
\hline EA at non-acupoint & - & - & + & + & + \\
\hline $\begin{array}{l}\text { EA at CV12 (low intensity } \\
\text { stimulation, } 10 \mathrm{~Hz} \text { ) }\end{array}$ & - & - & + & + & + \\
\hline Body weight and food intake & + & + & + & + & + \\
\hline Blood, duodenum, and brain stem & - & - & - & - & + \\
\hline
\end{tabular}

\subsection{Anorexia Model}

The rats were allowed to adapt to laboratory conditions for one week prior to the experiment. Anorexia was induced via a single intraperitoneal (i.p.) injection of $6 \mathrm{mg} / \mathrm{kg}$ cisplatin (saline was injected in the control group).

\subsection{Measurement of Body Weight and Daily Food Intake}

One day before the administration of cisplatin, the rats were placed individually in acrylic metabolic cages (JD-C-66; Jeung-Do Bio and Plant Co., Ltd., Seoul, Korea) until the end of the experiment. The body weight, food intake, water intake, and urine output were measured every $24 \mathrm{~h}$. Food intake was calculated as the difference between the food provided initially and the unconsumed food.

\subsection{Electro-acupuncture Treatment}

The CV12 acupuncture point was selected and located according to the acupuncture atlas for rats [15]. Disposable stainless-steel acupuncture needles $(0.25 \times 40 \mathrm{~mm}$; Dongbang Acupuncture Inc., Seoul, Korea) were inserted to a depth of $2 \mathrm{~mm}$ in the center of the abdominal midline and $1 \mathrm{~cm}$ below. The acupuncture point was stimulated using an EA system (ES-160; ITO Physiotherapy and Rehabilitation Co., Ltd., Tokyo, Japan) for $10 \mathrm{~min}$ at the indicated intensity and frequency. The peak amplitude applied was $16 \mathrm{~mA}$ for the low intensity stimulation and $32 \mathrm{~mA}$ the for high intensity stimulation. The frequency applied for the low and high stimulation were $10 \mathrm{~Hz}$ and $100 \mathrm{~Hz}$ respectively. During the EA process, the rats were anesthetized with $2.8 \%$ isoflurane (Hana Pharm 
Co., Ltd., Hwaseong, Korea), using oxygen as a carrier gas, at a flow rate of $400 \mathrm{~mL} / \mathrm{min}$. Inhalational anesthetics were provided mechanically (Parkland Scientific Inc., Coral Springs, FL, USA).

\subsection{Quantitative Analysis of Monoamine Neurotransmitters in Plasma}

Blood samples were collected from the heart of the anesthetized rats into a tube containing EDTA anticoagulant. After centrifugation $\left(1500 \times g, 4{ }^{\circ} \mathrm{C}\right.$ for $\left.10 \mathrm{~min}\right), 3.6 \mu \mathrm{L}$ of $70 \%$ perchloric acid was added to $100 \mu \mathrm{L}$ of the plasma supernatant. The mixture was centrifuged $\left(3000 \times g, 4{ }^{\circ} \mathrm{C}\right.$ for $10 \mathrm{~min}$ ) and filtered through a $0.22 \mu \mathrm{m}$ membrane filter. Further, $20 \mu \mathrm{L}$ of the filtrate was injected into a Nova-Pak C18 column (60 ̊, $4 \mu \mathrm{m}, 3.9 \mathrm{~mm} \times 150 \mathrm{~mm}$; Waters Corp., Milford, MA, USA) and analyzed using HPLC [16]. The flow rate of the mobile phase $(85 \mathrm{mM}$ citrate, $100 \mathrm{mM}$ sodium acetate, $0.9 \mathrm{mM}$ sodium octyl sulfate, $0.2 \mathrm{mM}$ EDTA, and $12 \%$ methanol, adjusted to $\mathrm{pH} 3.7$ ) was $1.0 \mathrm{~mL} / \mathrm{min}$. Monoamine neurotransmitters serotonin (5-hydroxytryptamine, 5-HT), 5-hydroxyindoleacetic acid (5-HIAA), dopamine (DA), and norepinephrine (NE) were detected and quantified by ECD (2465; Waters Corp., Milford, MA, USA).

\subsection{Measurement of Gastrointestinal Hormone Levels in the Plasma}

Blood samples were mixed with EDTA after collection to prevent coagulation. They were centrifuged immediately $\left(1500 \times \mathrm{g}, 4^{\circ} \mathrm{C}\right.$ for $\left.10 \mathrm{~min}\right)$ to obtain a supernatant. The plasma samples were stored at $-80{ }^{\circ} \mathrm{C}$ until required. The levels of GHRL and CCK were determined using ELISA kits (EK-069-04 and MM-402 respectively; Mitsubishi Kagaku Iatron Inc., Tokyo, Japan) according to the manufacturer's instructions.

\section{8. $R T-q P C R$}

The duodenum was dissected to explore the mRNA expression of GHRL by RT-qPCR and the hypothalamus was harvested for the detection of NPY and POMC (pro-opiomelanocortin). The total RNA was extracted from tissues using the Tri-RNA reagent (Favorgen Biotech Corp., Kaohsiung, Taiwan) following which, cDNA was synthesized using a RevertAid First Strand cDNA Synthesis Kit (Thermo Fisher Scientific Inc., Waltham, MA, USA) according to the manufacturer's protocols [17]. Reaction mixtures were prepared with cDNA and PowerUp SYBR Green Master Mix (Thermo Fisher Scientific Inc.) and a set of primers for the selected genes. The specific sequences of the primers are listed in Table 2. The qPCR was conducted using a QuantStudio 3 Real-Time PCR System (Thermo Fisher Scientific Inc.) applying the following conditions: initial denaturation at $95^{\circ} \mathrm{C}$ for $10 \mathrm{~min}$; 45 cycles of annealing at $95^{\circ} \mathrm{C}$ for $3 \mathrm{~s}$, and $59^{\circ} \mathrm{C}$ for $30 \mathrm{~s}$. The relative mRNA expression level for each gene was analyzed using the $2-\Delta \Delta \mathrm{Cq}$ method. $\beta$-actin was used as a reference gene.

Table 2. Primers used for RT-qPCR. GHRL— ghrelin; POMC—pro-opiomelanocortin; NPY: neuropeptide Y.

\begin{tabular}{ccc}
\hline Gene & Forward $\left(\mathbf{5}^{\prime} \rightarrow \mathbf{3}^{\prime}\right)$ & \multicolumn{1}{c}{ Reverse $\left(\mathbf{5}^{\prime} \rightarrow \mathbf{3}^{\prime}\right)$} \\
\hline GHRL & AGCCCAGCAGAGAAAGGAAT & GTGGCTGCAGTTTAGCTGGT \\
NPY & TGTCTCAGGGCTGGATCTCT & TACTCCGCTCTGCGACACTA \\
POMC & GCTTCATGACCTCCGAGAAG & TCTTGATGATGGCGTTCTTG \\
$\beta$-actin & AAGTCCCTCACCCTCCCAAAAG & AAGCAATGCTGTCACCTTCCC \\
\hline
\end{tabular}

\subsection{Western Blotting Analysis}

The brain stem was dissected and the NTS cells isolated, to detect c-Fos expression using the western blot technique. Tissues were homogenized in a non-ionic detergent buffer (1\% Nonidet-P40, $5 \%$ glycerol, $1 \mathrm{mM}$ EDTA, $25 \mathrm{mM}$ Tris- $\mathrm{HCl}$, and $150 \mathrm{mM} \mathrm{NaCl}$ at $\mathrm{pH}$ 7.5) and supplemented with a protease inhibitor mixture (Roche Co., Basel, Switzerland) for $20 \mathrm{~min}$ at $4{ }^{\circ} \mathrm{C}$. The lysates were centrifuged $\left(21,130 \times \mathrm{g}\right.$ at $4{ }^{\circ} \mathrm{C}$ for $\left.10 \mathrm{~min}\right)$ to obtain a protein supernatant. The protein concentration of each whole-cell extract was determined using the Bio-Rad Protein Assay Kit (Bio-Rad Laboratories Inc., 
Hercules, CA, USA). Equal amounts of protein from each protein extract ( $30 \mu \mathrm{g} / \mathrm{lane})$ were separated by electrophoresis on an $8 \%$ sodium dodecyl sulfate-polyacrylamide gel and blotted onto PVDF transfer membranes. Epitope-specific primary antibodies including c-Fos and $\alpha$-actin conjugated with secondary antibodies (Cell Signaling Technology Inc., Danvers, MA, USA) were used to label the target proteins. The bound antibodies were visualized with Immobilon Western Chemiluminescent HRP Substrate (EMD Millipore Co., Billerica, MA, USA) and analyzed using a digital imaging system (LAS-4000; GE Healthcare Bio-Sciences Corp., Little Chalfont, UK).

\subsection{Statistical Analysis}

A non-parametric method, the Kruskal-Wallis test, was first performed to analyze variance. The multiple comparisons test was performed by using Dunn's pairwise test with Bonferroni adjustment as a post-hoc test. Further, the Mann-Whitney U test was used to compare the differences between experimental groups. All the statistical analyses were performed by SPSS Statistics 21.0 (IBM Corp., Armonk, NY, USA). A p-value less than 0.05 was considered statistically significant.

\section{Results}

\subsection{Evaluation of the Optimal EA Condition to Alleviate Cisplatin-Induced Anorexia}

The intensity and frequency of EA applied to stimulate acupuncture were modified and the therapeutic effect of each condition was assessed based on the changes in body weight and food intake between the experimental groups. The body weight of rats in all groups decreased one day after cisplatin was injected. In the test for the intensity of EA, the average weight was restored better in the group treated with EA at the intensity of 1 unit (Figure 1A) than the group treated with 4 units. The differences of average weight between day 0 and day 3 in each group were $-16.72 \mathrm{~g}$ for Cisplatin, $+4.73 \mathrm{~g}$ for Cisplatin + CV12 (1 unit), and $-3.57 \mathrm{~g}$ for Cisplatin + CV12 (4 unit). Both EA groups also expressed improved nutritional tolerance after treatment with cisplatin.

The optimal frequency for EA was also studied. Stimulating EA at $10 \mathrm{~Hz}$ was seen to restore the body weight in the rats, whereas treatment at $100 \mathrm{~Hz}$ suppressed the recovery of the body weight even more than that observed in the control group (Figure 1B). The decrease in average weight between day 0 and day 3 was $10.62 \mathrm{~g}$ for Cisplatin, $2.52 \mathrm{~g}$ for Cisplatin + CV12 (10 Hz), and $17.13 \mathrm{~g}$ for Cisplatin + CV12 $(100 \mathrm{~Hz})$. Significant differences in food intake were not observed between the Cisplatin and the Cisplatin + CV12 $(10 \mathrm{~Hz})$ groups. In the Cisplatin + CV12 $(100 \mathrm{~Hz})$ group, the intake of food decreased.

Based on these results, the optimal condition for EA treatment was defined as 1 unit for intensity and $10 \mathrm{~Hz}$ for frequency. The anti-anorexic effect was reconfirmed using the same model. On treatment with EA (1 unit, $10 \mathrm{~Hz}$ ), the body weight of the rats recovered better than the group treated only with cisplatin (Figure 1C). The reduction in average weight between day 0 and day 3 in each group was $29.83 \mathrm{~g}$ for Cisplatin and $10.05 \mathrm{~g}$ for Cisplatin + CV12 (1 unit, $10 \mathrm{~Hz})$. EA treatment also improved food intake after the end of the experimental period. 
(A)

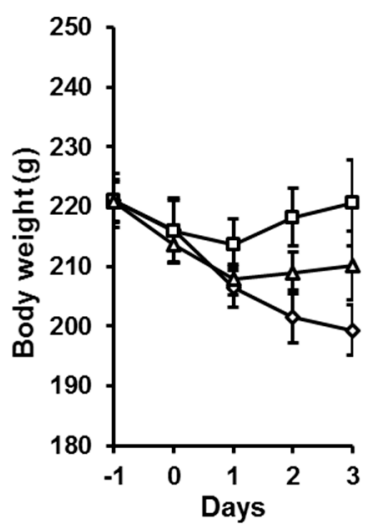

(B)

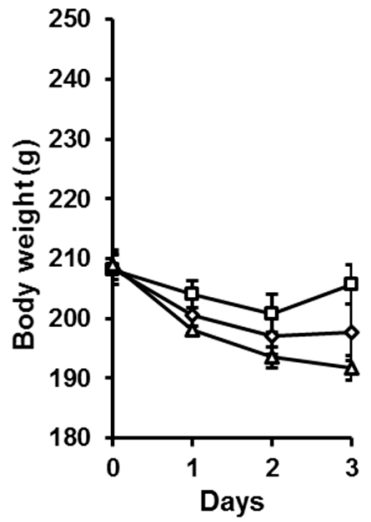

(C)

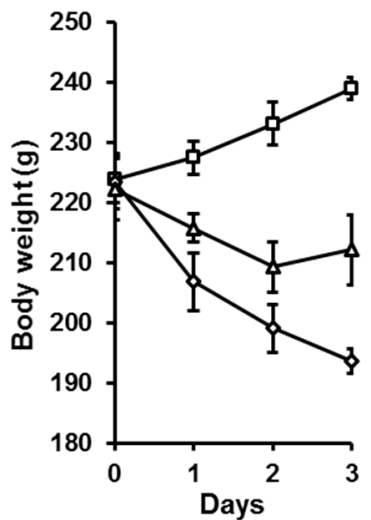

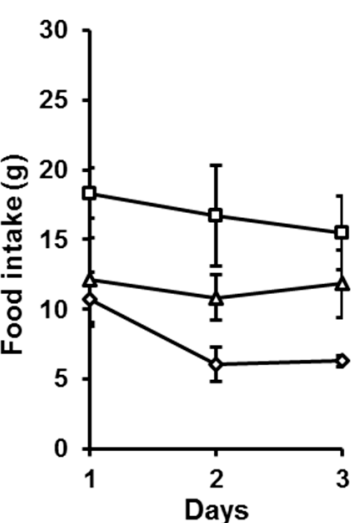

- Cisplatin

Cisplatin + CV12 (1 unit)

$\Delta$ Cisplatin + CV12 (4 units)

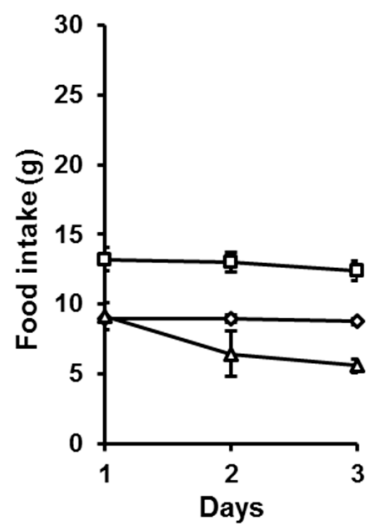

- Cisplatin

- Cisplatin + CV12 (10 Hz)

$\Delta$ Cisplatin + CV12 $(100 \mathrm{~Hz})$

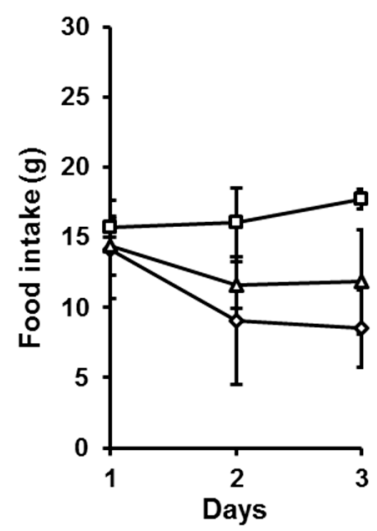

- Cisplatin

$\square$ Vehicle

$\Delta$ Cisplatin + CV12

(1 unit, $10 \mathrm{~Hz}$ )

Figure 1. Changes in the body weight and food intake of rats during the experimental period. (A) Optimal intensity of EA, $(n=3)$ : Cisplatin (injected with cisplatin, without EA), Cisplatin + CV12 (1 unit) (injected with cisplatin, EA at the intensity of 1 unit), and Cisplatin + CV12 (4 units) (injected with cisplatin, EA at the intensity of 4 units). (B) Optimal frequency of EA, $(n=3)$ : Cisplatin (injected with cisplatin, without EA), Cisplatin + CV12 $(10 \mathrm{~Hz})$ (injected with cisplatin, EA at the frequency of $10 \mathrm{~Hz})$, and Cisplatin + CV12 $(100 \mathrm{~Hz})$ (injected with cisplatin, EA at the frequency of $100 \mathrm{~Hz}$ ). (C) Confirmation of the anti-anorexic effect of the chosen optimal conditions for EA (1 unit, $10 \mathrm{~Hz}$ ), $(n=3)$ : Vehicle (injected with saline, EA at the non-acupoint with the intensity of 1 unit and frequency of $10 \mathrm{~Hz}$ ), Cisplatin (injected with cisplatin, without EA), and Cisplatin + CV12 (injected with cisplatin, EA at the CV12 acupoint with the intensity of 1 unit and frequency of $10 \mathrm{~Hz}$ ). $p<0.05$ was considered statistically significant.

\subsection{Plasma Monoamine Neurotransmitters' Concentrations}

On treatment with cisplatin, the concentrations of 5-HT, 5-HIAA, DA, and NE in the plasma were significantly higher in the test group than the control group. EA stimulation at the CV12 acupoint reduced the levels of these plasma monoamine neurotransmitters, especially that of NE. The HPLC 
chromatograms and corresponding quantitative graphs of monoamine neurotransmitters are presented in Figure 2.

(A)
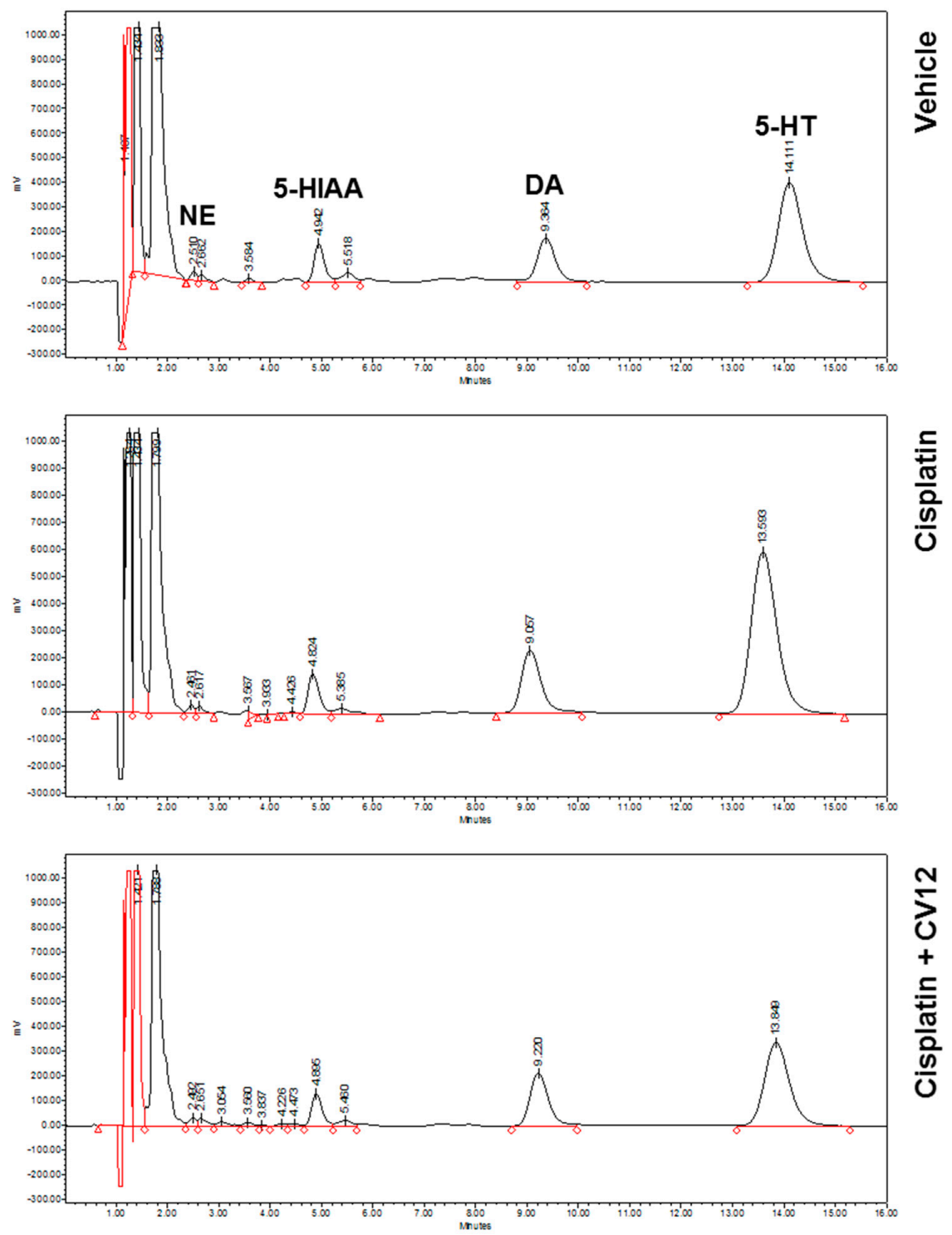

(B)

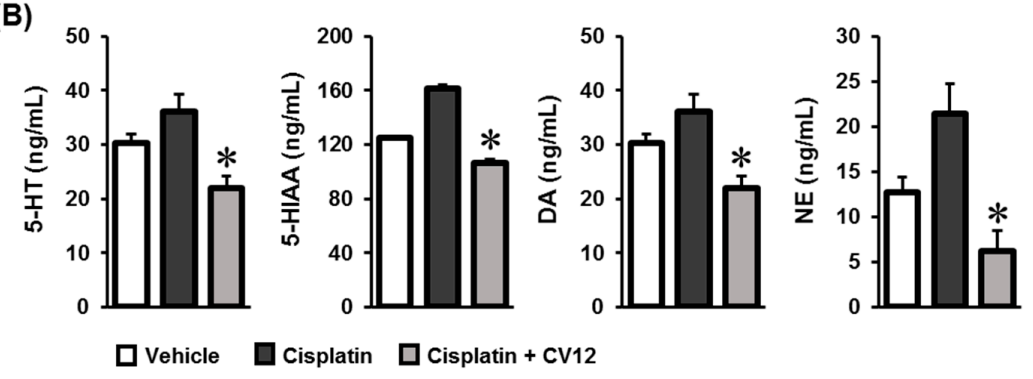

Figure 2. Quantitative analysis of monoamine neurotransmitters in plasma. The concentrations of 5-HT, 5-HIAA, dopamine (DA), and norepinephrine (NE) were quantitatively analyzed by an HPLC-ECD system. (A) The HPLC chromatograms. (B) The quantitative graphs. ${ }^{*} \mathrm{p}<0.05$ was considered statistically significant. (5-HT: 5-hydroxytryptamine; 5-HIAA: 5-hydroxyindoleacetic acid; DA: dopamine; NE: norepinephrine). 


\subsection{Gastrointestinal Hormone Levels in Plasma}

The gastrointestinal hormone levels in plasma were measured using ELISA kits. The results are shown in Figure 3. The level of GHRL slightly increased in the EA-treated group compared with other groups. There were no statistical differences in the levels of CCK across the experimental groups.

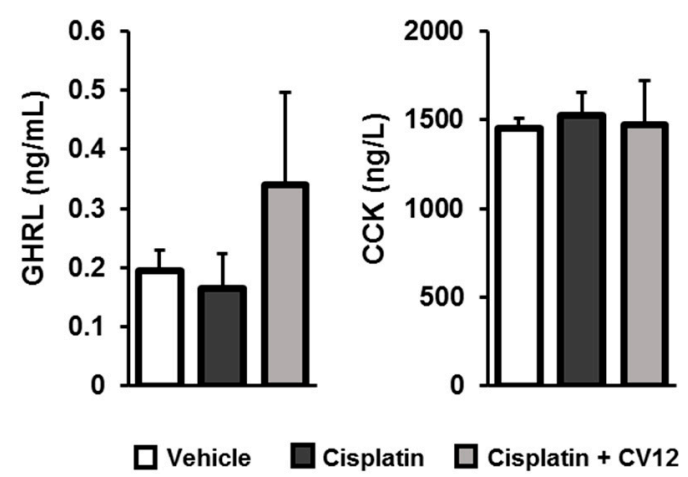

Figure 3. Gastrointestinal hormone levels in plasma. The levels of plasma GHRL and CCK were determined using ELISA kits. (GHRL: ghrelin; CCK: cholecystokinin).

\section{4. mRNA Expression Levels of GHRL, NPY, and POMC}

RT-qPCR was conducted to detect the mRNA expression levels of GHRL, NPY, and POMC. Upon treatment with EA at CV12, the expression of GHRL was stimulated in the duodenum. The expression of NPY from the hypothalamus slightly increased while that of POMC showed no change between experimental groups. The results of RT-qPCR are depicted in Figure 4.
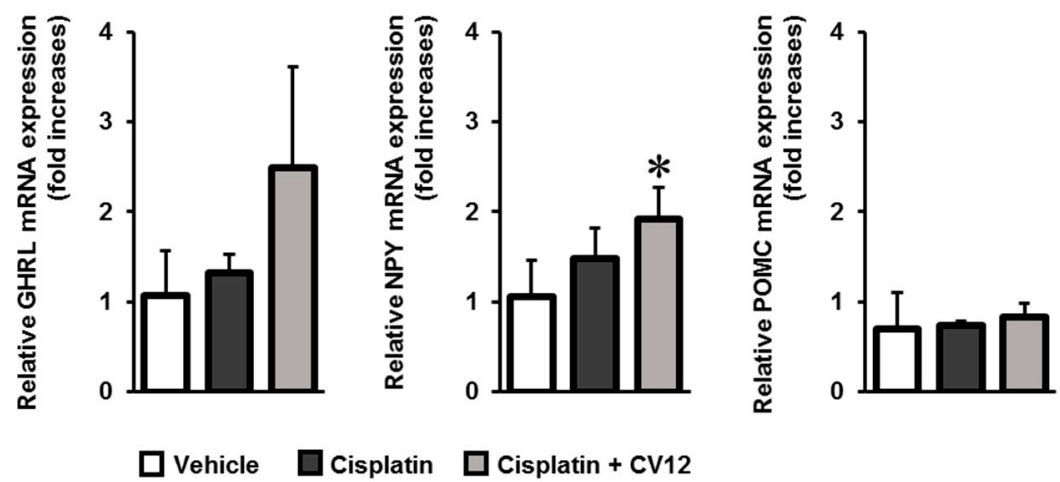

Figure 4. The mRNA expression levels of GHRL, NPY, and POMC were evaluated by RT-qPCR. * $p<0.05$ was considered statistically significant. (GHRL: ghrelin; NPY: neuropeptide Y; POMC: pro-opiomelanocortin).

\section{5. c-Fos Expression in the NTS}

The protein expression of c-Fos in NTS cells was detected using western blot. The results showed no significant changes between experimental groups (Figure 5). 


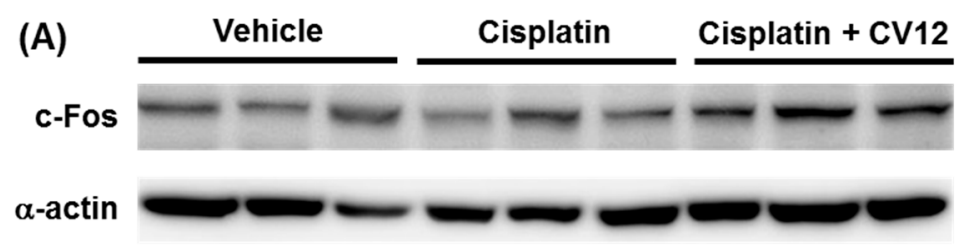

(B)

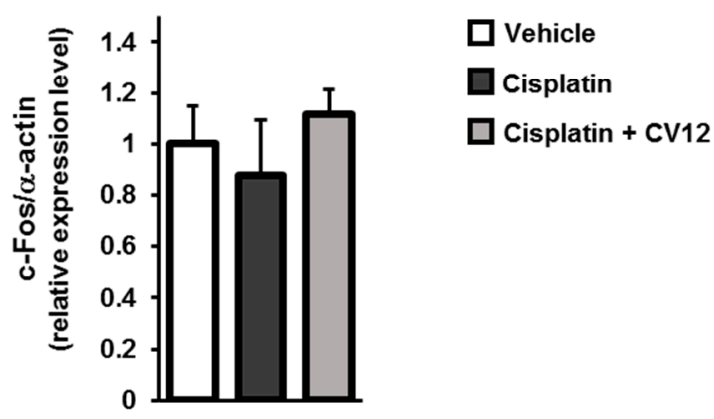

Figure 5. c-Fos expression in NTS cells. (A) Images of western blot analysis for the expression of c-Fos and $\alpha$-actin. (B) The quantitative graph of c-Fos expression with $\alpha$-actin as a loading control.

\section{Discussion}

Previous studies have compared the anti-anorexic effects of EA stimulation in different acupuncture points, including CV12, PC6, and ST36. Among these, CV12 was assessed as the most effective acupoint based on the food intake and recovery in body weight of the experimental models [14]. CV12, also known as Zhongwan, has been used in many research studies and clinical trials. EA at CV12 expressed an anti-emetic effect in rats by reducing the level of 5-HT in the duodenum and suppressing the expression of c-Fos in the NTS [12]. EA at ST25 and CV12 alleviated visceral pain in rats with post-inflammatory irritable bowel syndrome by inhibiting enterochromaffin cells and reducing the concentration of 5-HT [18]. Additionally, the therapeutic effect of acupuncture stimulation at CV12 for persistent hiccups [19] and pain in patients with cancer [20] was clinically studied. Hence the CV12 acupoint was chosen to study the optimal intensity and frequency for EA, followed by pharmacological mechanism studies. It was found that the optimal condition for EA stimulation to reduce cisplatin-induced anorexia in rats was $1 \mathrm{unit}$ for the intensity and $10 \mathrm{~Hz}$ for the frequency. The effects on monoamine neurotransmitters, gastrointestinal hormones, and NTS cells were evaluated by molecular studies.

The monoamine neurotransmitters 5-HT, DA, NE, and epinephrine are involved in multiple functions such as the control of respiratory, cardiovascular, gastrointestinal, and psychomotor activities along with the regulation of hormone secretion, sleep, body temperature, and pain [21]. Serotonin, or 5-HT, affects satiety by activating multiple serotonergic receptors with discrete roles [22,23]. Studies performed in animal models have determined that 5-HT, at a high concentration, reduces total energy intake [24,25]. A major metabolite of 5-HT, 5-HIAA, has been used as an indicator for 5-HT activity in the central nervous system and the peripheral nervous system. The levels of 5-HT, 5-HIAA, DA, and NE in the plasma of experimental rats were quantified by HPLC-ECD analysis. Increased levels of 5-HT, 5-HIAA, DA and NE might exert metabolic effects contributing to the impairment characteristics for anorexia. The results obtained suggested that after cisplatin injection increased the concentrations of these plasma monoamine neurotransmitters, EA stimulation at CV12 was able to reduce them. This suggests that the therapeutic effect of EA at CV12 against cisplatin-induced anorexia in rats affects the signaling pathways of the serotonergic system.

Aside from monoamine neurotransmitters, gastrointestinal hormones and neuropeptides also participate in the regulation of appetite. GHRL is a key peptide in the gut that controls food intake and energy expenditure through its orexigenic effect. GHRL increases food intake in rats and stimulates the expression of NPY in the hypothalamus [26]. In contrast, CCK is the anorexigenic gastrointestinal hormone that causes a reduction in the meal size consumed and the duration of feeding [27]. Plasma 
GHRL levels were increased by EA treatment in the cisplatin-induced anorexic rats. The mRNA expression of GHRL in the duodenum tissue was also significantly stimulated after EA treatment. EA stimulation at CV12 did not cause any considerable difference in the concentration of plasma CCK between the experimental groups.

Hypothalamic neuropeptides act as key signaling agents in regulating appetite and feeding behaviors. NPY and orexin (also known as hypocretin) are orexigenic neuropeptides which stimulate feeding; whereas POMC, an $\alpha$ melanocyte-stimulating hormone, and corticotrophin-releasing hormone are considered anorexigenic neuropeptides [28]. The pathophysiological anorexic animal model illustrated that cisplatin reduced the gene expression of NPY, but enhanced the expression of POMC in the arcuate nucleus of the hypothalamus in rats [29]. EA stimulation increased the mRNA expression of NPY in the hypothalamus but did not affect the expression of POMC. The expression of c-Fos in NTS, a mediator of several neurotransmitter signaling pathways [30,31], was investigated but no significant changes occurred. Essentially, the anti-anorexic effect of EA treatment may cause the stimulation of GHRL which leads to up-regulation of NPY expression to improve appetite.

Our studies suggested that the body weight and appetite of rats significantly recovered by treatment with electro-acupuncture at CV12. The EA stimulation also induced changes in the levels of hormones and neurotransmitters related to appetite controlling. The acupuncture point CV12 has been studied in many clinical trials for the prevention of nausea and vomiting induced by chemotherapy [32-35]. In addition, the acupuncture treatment at CV12 showed a significant improvement in persistent hiccups [19] and pains [20] in patients with cancer.

\section{Conclusions}

Electro-acupuncture was used to improve the side effects of chemotherapy, including nausea, insomnia, cachexia, and pain, in patients with cancer. In this study, the optimal condition of EA to alleviate anorexia in rats was found to be 1 unit for intensity and $10 \mathrm{~Hz}$ for frequency. Pharmacological studies demonstrated that EA treatment at CV12 reduces the levels of plasma monoamine neurotransmitters 5-HT, 5-HIAA, DA, and NE; while simultaneously stimulating the expression of GHRL and NPY to alleviate cisplatin-induced anorexia in rats.

Author Contributions: K.S.K. and T.-H.K. conceived and designed the experiments; J.Y.B., T.A.T., J.H.S., H.Y.K., J.L., J.K., H.J.C., and W.H. performed the experiments; K.S.K., J.H.S., H.Y.K., and H.J.C. analyzed the data; T.A.T. and J.H.S. interpreted the data and contributed to manuscript structure and flow; T.A.T. and K.S.K. wrote the paper. All authors reviewed and confirmed the manuscript.

Funding: This study was supported by the Korea Health Technology R\&D Project through the Korea Health Industry Development Institute, funded by the Ministry of Health \& Welfare, Republic of Korea (grant no. HI15C0089). This study was also funded by a research grant from the National Research Foundation of Korea (2019R1F1A1059173).

Acknowledgments: In this section you can acknowledge any support given which is not covered by the author contribution or funding sections. This may include administrative and technical support, or donations in kind (e.g., materials used for experiments).

Conflicts of Interest: The authors declare no conflict of interest.

$\begin{array}{ll}\text { Abbreviations } \\ \text { 5-HIAA } & \text { 5-hydroxyindoleacetic acid } \\ \text { 5-HT } & \text { 5-hydroxytryptamine } \\ \text { CCK } & \text { cholecystokinin } \\ \text { DA } & \text { dopamine } \\ \text { EA } & \text { electro-acupuncture } \\ \text { ELISA } & \text { enzyme-linked immunosorbent assay } \\ \text { GHRL } & \text { ghrelin } \\ \text { HPLC-ECD } & \text { high performance liquid chromatography-electrochemical detection } \\ \text { i.p. } & \text { intraperitoneal }\end{array}$




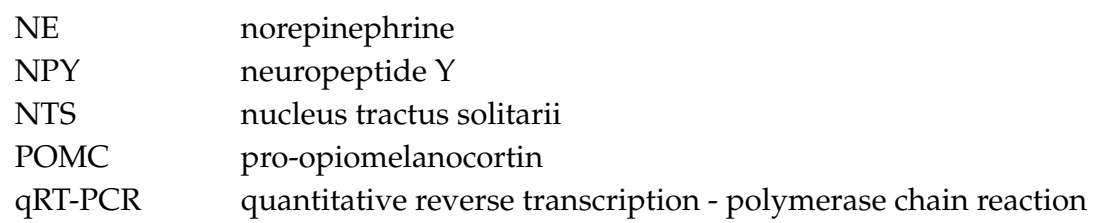

\section{References}

1. Jung, K.W.; Won, Y.J.; Kong, H.J.; Lee, E.S. Cancer Statistics in Korea: Incidence, Mortality, Survival, and Prevalence in 2016. Cancer Res. Treat. 2019, 51, 417-430. [CrossRef]

2. Jung, K.W.; Won, Y.J.; Kong, H.J.; Lee, E.S. Prediction of Cancer Incidence and Mortality in Korea, 2019. Cancer Res. Treat. 2019, 51, 431-437. [CrossRef]

3. Tisdale, M.J. Cancer anorexia and cachexia. Nutrition 2001, 17, 438-442. [CrossRef]

4. Laviano, A.; Koverech, A.; Seelaender, M. Assessing pathophysiology of cancer anorexia. Curr. Opin. Clin. Nutr. Metab. Care 2017, 20, 340-345. [CrossRef]

5. Muliawati, Y.; Haroen, H.; Rotty, L.A.W. Cancer anorexia-cachexia syndrome. Acta Med. Indones. Indones. J. Intern. Med. 2012, 44, 154-162.

6. Laviano, A.; Meguid, M.M.; Rossi-Fanelli, F. Cancer anorexia: Clinical implications, pathogenesis, and therapeutic strategies. Lancet Oncol. 2003, 4, 686-694. [CrossRef]

7. Laviano, A.; Meguid, M.M.; Inui, A.; Muscaritoli, M.; Rossi-Fanelli, F. Therapy insight: Cancer anorexiacachexia syndrome-When all you can eat is yourself. Nat. Rev. Clin. Oncol. 2005, 2, 158-165. [CrossRef]

8. Ezzo, J.; Richardson, R.M.; Vickers, A.; Allen, C.; Dibble, S.; Issell, B.F.; Lao, L.; Pearl, M.; Ramirez, G.; Roscoe, J.A.; et al. Acupuncture-point stimulation for chemotherapy-induced nausea or vomiting. Cochrane Database Syst. Rev. 2006. [CrossRef]

9. Jeanette Ezzo, K.S. Antonius Schneider, Cochrane systematic reviews examine P6 acupuncture-point stimulation for nausea and vomiting. J. Altern. Complemen. Med. 2006, 12, 489-495. [CrossRef]

10. Kim, M.; Kim, J.-E.; Kim, A.R.; Park, H.J.; Kwon, O.J.; Kim, B.K.; Cho, J.H.; Kim, J.H. Electroacupuncture for treating insomnia in patients with cancer: A study protocol for a randomised pilot clinical trial. BMJ Open 2017, 7, e016269. [CrossRef]

11. Chen, H.; Liu, T.Y.; Kuai, L.; Zhu, J.; Wu, C.J.; Liu, L.M. Electroacupuncture treatment for pancreatic cancer pain: A randomized controlled trial. Pancreatology 2013, 13, 594-597. [CrossRef] [PubMed]

12. Cui, Y.; Wang, L.; Shi, G.; Liu, L.; Pei, P.; Guo, J. Electroacupuncture alleviates cisplatin-induced nausea in rats. Acupunct. Med. 2016, 34, 120-126. [CrossRef] [PubMed]

13. Liu, Y.; Zhang, S.; Ye, F.; Yin, J.; Li, S.; Chen, J.D.Z. Ameliorating effects and mechanisms of chronic electroacupuncture at ST36 in a rodent model of dyspepsia induced by cisplatin. Neurogastroenterol. Motil. 2019, 31, e13474. [CrossRef] [PubMed]

14. Kang, K.S.; Huh, W.; Bang, Y.; Choi, H.J.; Baek, J.Y.; Song, J.H.; Kang, J.W.; Kim, T.H. Electroacupuncture for chemotherapy-induced anorexia through humoral appetite regulation: A preliminary experimental study. Exp. Ther. Med. 2019, 17, 2587-2597. [CrossRef]

15. Li, Z.R. Experimental Acupuncture Science; China Press of Traditional Chinese Medicine: Beijing, China, 2003.

16. Park, R.; Lee, K.I.; Kim, H.; Jang, M.; Ha, T.K.Q.; Oh, W.K.; Park, J. Reserpine treatment activates AMP activated protein kinase (AMPK). Nat. Prod. Sci. 2017, 23, 157-161. [CrossRef]

17. Yoon, D.H.; Han, C.; Fang, Y.; Gundeti, S.; Han Lee, I.-S.; Song, W.O.; Hwang, K.-C.; Kim, T.W.; Sung, G.-H.; Park, H. Inhibitory activity of cordyceps bassiana extract on LPS-induced inflammation in RAW 264.7 cells by suppressing NF-kB activation. Nat. Prod. Sci. 2017, 23, 162-168. [CrossRef]

18. Zhu, X.; Liu, Z.; Qin, Y.; Niu, W.; Wang, Q.; Li, L.; Zhou, J. Analgesic effects of electroacupuncture at ST25 and CV12 in a rat model of postinflammatory irritable bowel syndrome visceral pain. Acupunct. Med. 2018, 36, 240-246. [CrossRef]

19. Ge, A.X.; Ryan, M.E.; Giaccone, G.; Hughes, M.S.; Pavletic, S.Z. Acupuncture treatment for persistent hiccups in patients with cancer. J. Altern. Complement. Med. 2010, 16, 811-816. [CrossRef]

20. Kim, K.; Lee, S. Intradermal Acupuncture Along with Analgesics for Pain Control in Advanced Cancer Cases: A Pilot, Randomized, Patient-Assessor-Blinded, Controlled Trial. Integr. Cancer Ther. 2018, 17, 1137-1143. [CrossRef] 
21. Ng, J.; Papandreou, A.; Heales, S.J.; Kurian, M.A. Monoamine neurotransmitter disorders-clinical advances and future perspectives. Nat. Rev. Neurol. 2015, 11, 567-584. [CrossRef]

22. Beaudoin-Gobert, M.; Sgambato-Faure, V. Serotonergic pharmacology in animal models: From behavioral disorders to dyskinesia. Neuropharmacology 2014, 81, 15-30. [CrossRef] [PubMed]

23. Kim, M.H.; Lee, J.; Jung, S.; Kim, J.W.; Shin, J.H.; Lee, H.J. The involvement of ginseng berry extract in blood flow via regulation of blood coagulation in rats fed a high-fat diet. J. Ginseng Res. 2017, 41, 120-126. [CrossRef] [PubMed]

24. Lam, D.D.; Przydzial, M.J.; Ridley, S.H.; Yeo, G.S.; Rochford, J.J.; O’Rahilly, S.; Heisler, L.K. Serotonin 5-HT2C receptor agonist promotes hypophagia via downstream activation of melanocortin 4 receptors. Endocrinology 2008, 149, 1323-1328. [CrossRef] [PubMed]

25. Cambraia, R.P.B. Psychobiological aspects of feeding behavior. Rev. Nutr. 2004, 17, 217-225. [CrossRef]

26. Ramos, E.J.B.; Suzuki, S.; Marks, D.; Inui, A.; Asakawa, A.; Meguid, M.M. Cancer anorexia-cachexia syndrome: Cytokines and neuropeptides. Curr. Opin. Clin. Nutr. Metab. Care 2004, 7, 427-434. [CrossRef]

27. Yu, J.H.; Kim, M.S. Molecular mechanisms of appetite regulation. Diabetes Metab. J. 2012, 36, $391-398$. [CrossRef]

28. Yoichi Ueta, H.H. Etsuro Onuma, Yoh Takuwa, and Etsuro Ogata, Hypothalamic neuropeptides and appetite response in anorexia-cachexia animal. Endocr. J. 2007, 54, 831-838. [CrossRef]

29. Yoshimura, M.; Matsuura, T.; Ohkubo, J.; Ohno, M.; Maruyama, T.; Ishikura, T.; Hashimoto, H.; Kakuma, T.; Yoshimatsu, H.; Terawaki, K.; et al. The gene expression of the hypothalamic feeding-regulating peptides in cisplatin-induced anorexic rats. Peptides 2013, 46, 13-19. [CrossRef]

30. Holland, R.A.; Leonard, J.J.; Kensey, N.A.; Hannikainen, P.A.; De Jonghe, B.C. Cisplatin induces neuronal activation and increases central AMPA and NMDA receptor subunit gene expression in mice. Physiol. Behav. 2014, 136, 79-85. [CrossRef]

31. Lee, J.H.; Min, D.S.; Lee, C.W.; Song, K.H.; Kim, Y.S.; Kim, H.P. Ginsenosides from Korean Red Ginseng ameliorate lung inflammatory responses: Inhibition of the MAPKs/NF-kB/c-Fos pathways. J. Ginseng Res. 2018, 42, 476-484. [CrossRef]

32. Chen, B.; Hu, S.; Liu, B.; Zhao, T.; Li, B.; Liu, Y.; Li, M.; Pan, X.; Guo, Y.; Chen, Z.; et al. Efficacy and safety of electroacupuncture with different acupoints for chemotherapy-induced nausea and vomiting: Study protocol for a randomized controlled trial. Trials 2015, 16, 212. [CrossRef] [PubMed]

33. Gao, L.; Chen, B.; Zhang, Q.; Zhao, T.; Li, B.; Sha, T.; Zou, J.; Guo, Y.; Pan, X.; Guo, Y. Acupuncture with different acupoint combinations for chemotherapy-induced nausea and vomiting: Study protocol for a randomized controlled trial. BMC Complement. Altern. Med. 2016, 16, 441. [CrossRef] [PubMed]

34. Chen, B.; Guo, Y.; Zhao, X.; Gao, L.L.; Li, B.; Zhao, T.Y.; Zhang, Q.W.; Zou, J.X.; Li, M.Y.; Guo, Y.M.; et al. Efficacy differences of electroacupuncture with single acupoint or matching acupoints for chemotherapy-induced nausea and vomiting: Study protocol for a randomized controlled trial. Trials 2017, 18, 477. [CrossRef] [PubMed]

35. Li, Q.; Yu, M.; Yang, G.; Wang, X.; Wang, H.; Zhang, C.; Xue, N.; Xu, W.; Fu, Q.; Yang, Z.; et al. Effect of acupuncture in prevention and treatment of chemotherapy-induced nausea and vomiting in patients with advanced cancer: Study protocol for a randomized controlled trial. Trials 2017, 18, 185. [CrossRef]

(C) 2019 by the authors. Licensee MDPI, Basel, Switzerland. This article is an open access article distributed under the terms and conditions of the Creative Commons Attribution (CC BY) license (http://creativecommons.org/licenses/by/4.0/). 